\title{
Reologia e Compactação de Pós Atomizados para Porcelanatos de Grande Tamanho
}

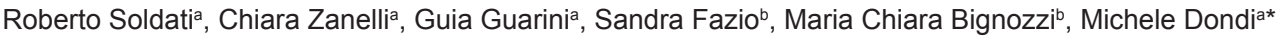 \\ ${ }^{a}$ Consiglio Nazionale delle Ricerche - CNR, Istituto di Scienza e Tecnologia dei Materiali Ceramici - ISTEC, Faenza, Itália \\ ${ }^{b}$ Centro Cerâmico de Bolonha, Bologna, Itália \\ *e-mail: michele.dondi@istec.cnr.it
}

\begin{abstract}
Resumo
O comportamento tecnológico de amostras de porcelanato durante o carregamento e prensagem de grandes formatos depende das propriedades reológicas dos pós atomizados e de como a compactação as afeta. Apesar de que as informações publicadas dão uma ideia das características dos pós para placas cerâmicas secos por atomização, não há dados disponíveis sobre os suportes produzidos por tecnologias inovadoras para grandes formatos $\left(>4 \mathrm{~m}^{2}\right)$. Desta forma, foi realizado um estudo sistemático das propriedades e do comportamento de pós secos por atomização para grandes formatos. Foram caracterizados 11 pós atomizados industriais segundo suas características intrínsecas (distribuição de tamanho de partículas e de aglomerado, distribuição de forma e umidade em função do tamanho do aglomerado); as propriedades reológicas (fluxo mássico, ângulos estáticos e dinâmicos de repouso, densidade aparente sem compactar e após compactação); comportamento em compactação (curvas de densidade aparente, porosidade intergranular e intragranular em função da carga aplicada); e comportamento na queima, com objetivo de mostrar qualquer efeito de densidade aparente a seco sobre a contração e da densidade aparente sobre as amostras queimadas. Foi observado o efeito destas características intrínsecas sobre a fluência e compressão dos pós, bem como as relações mútuas entre os parâmetros reológicos. Foram produzidas duas classes amplas de pós atomizados com uma distribuição de tamanho de aglomerado mais fina e mais grossa. Os resultados mostram que os pós cerâmicos fluem livremente, com propriedades reológicas que variam em um intervalo bastante estreito, o que dificulta estabelecer correlações estritas entre os diferentes métodos. A fluência depende, em grande medida, da aparição de agregados mais grossos, com forma irregular, que derivam da coalescência de três ou mais aglomerados separados. As características das peças cruas afetam as amostras queimadas, em particular no que se refere à cinética de densificação e à contração, embora as diferenças iniciais diminuam durante a queima. O rendimento das amostras secas por atomização, durante a compactação, é essencial para controlar a uniformidade da porosidade e da densidade aparente, repercutindo nas propriedades finais das placas. As tensões residuais podem estar relacionadas às contrações diferenciais durante a queima, causadas por gradientes de densidade.
\end{abstract}

Palavras-chave: porcelanato; grandes formatos; reologia de pós; pós atomizados.

\section{Introdução}

A forma como os pós atomizados fluem ao sair do carro alimentador e se depositam em uma matriz ou em uma correia afeta a estrutura das placas cruas, principalmente sua densidade aparente e a resistência mecânica $[1,2]$. Este comportamento depende das características dos pós atomizados, como a distribuição do tamanho do aglomerado, a forma dos grânulos e a distribuição da umidade em função do tamanho do aglomerado.

Desta forma, existe um preocupação crescente no setor cerâmico com as propriedades reológicas dos pós atomizados que possam determinar adequadamente seu comportamento durante o carregamento e a compactação, principalmente para grandes formatos [3, 4]. A reologia do pó pode ser medida segundo distintos métodos [5-7]. Entre eles estão: ângulo estático de repouso [8-11]; ângulo dinâmico de repouso [12]; densidade aparente sem compactação, densidade após compactação e índice de Hausner [8, 11, 13, 14]; e fluxo de massa através de um orifício [6].

A compactação do pó é um passo chave na fabricação de placas cerâmicas. Rege a distribuição espacial e quantitativa da porosidade em produtos crus e semiacabados secos. Estas características controlam a resistência mecânica das placas não queimadas, além da contração na queima e a microestrutura final em relação à porosidade residual $[15,16]$. Isto ocorre para grandes formatos obtidos com tecnologias inovadoras de compactação $[3,4,17]$ e em placas ou muito finas ou muito espessas $[18,19]$. O comportamento na prensagem de corpos cerâmicos depende das características dos pós atomizados (distribuição do tamanho do aglomerado, forma do grânulo e resistência mecânica, porosidade) e da forma como a matriz é carregada (ou se deposita sobre a esteira) e como os grânulos se reorganizam sob uma pressão crescente $[20,21]$. 
Pouca atenção foi dada às características dos pós atomizados pelo setor cerâmico: em primeiro lugar, ao grés de pasta vermelha $[22,23]$ e, em seguida, ao porcelanato [24, 25]. Porém não existe um panorama do comportamento reológico dos pós atomizados utilizados na fabricação de porcelanato de grande formato. Por outro lado, o comportamento em compactação de pós atomizados desde sempre é um objetivo na pesquisa de cerâmica avançada $[20,21,26,27]$ bem como de placas cerâmicas $[1,2,22,28]$. O interesse pela pesquisa em porcelanato tem aumentado na última década [2, 24, 25, 29-32]. As informações publicadas mostram dados sobre a distribuição de tamanho do aglomerado, a porosidade, a resistência mecânica e a microestrutura de pós atomizados junto às curvas de compactação e às características das placas prensadas, p.ex., densidade aparente, anisotropia, microestrutura, distribuição de tamanho de poro, e sua dependência em relação aos parâmetros de processamento.

O objetivo deste estudo preliminar é descrever as características reológicas principais de pastas industriais e fazer uma descrição do comportamento de compactação de pós atomizados usados para a fabricação de placas grandes de porcelanato. Os objetivos são: a) entender que parâmetros são significativos no caso de porcelanatos de formato grande, e b) construir uma base de dados para modelar o comportamento reológico dos pós cerâmicos atomizados.

\section{Procedimento Experimental}

Foram selecionadas 11 amostras de pós atomizados de porcelanato usados na fabricação de placas de grade formato, que foram caracterizadas por suas propriedades físicas e reológicas. As características determinadas foram: distribuição de tamanho de aglomerado por peneiramento a seco (malhas de 1 e 0,1 mm), forma dos grânulos (microscopia óptica de frações peneiradas com análise modal de micrografias) e distribuição da umidade em função do tamanho do aglomerado (diferença de massa de frações úmidas e secas para cada fração peneirada).

Foi pesquisada a reologia do pó pela determinação de (Figura 1): ângulo estático de repouso, $\alpha_{\text {est }}$ [9], e ângulo dinâmico de repouso, $\alpha_{\text {din }}[12]$; densidade aparente sem compactação $\left(\rho_{\text {ap }}\right)$, densidade compactada $\left(\rho_{\text {comp }}\right)$ e índice de Hausner, IH [8]; índice de fluxo mássico $\left(\mathrm{I}_{\mathrm{fm}}\right)$ com o uso de um copo Ford.

Foi pesquisado o comportamento em compactação em função da pressão uniaxial até aproximadamente $800 \mathrm{kgf} / \mathrm{cm}^{2}$ (80 MPa) com prensas hidráulicas de laboratório. Os moldes tinham uma dimensão $\mathrm{L}_{\mathrm{m}}$ de $50 \mathrm{~mm}$ (diâmetro) e de $110 \times 55 \mathrm{~mm}$. As amostras prensadas foram caracterizadas medindo-se sua massa e suas dimensões (largura $\times$ comprimento $\times$ espessura $=$ volume $)$. A densidade aparente a cru foi determinada pelo índice de massa/volume. O índice de compressibilidade de Carr foi calculado como $\mathrm{IC}=100\left(\mathrm{~V}_{\mathrm{m}}-\mathrm{V}_{\mathrm{p}}\right) / \mathrm{V}_{\mathrm{p}}$, onde $\mathrm{V}_{\mathrm{m}}$ é o volume da matriz preenchida com o pó e $V_{p}$ é o volume da amostra prensada.

A evolução microestrutural foi determinada pela observação da superfície normal em relação ao eixo do compacto, em um microscópio óptico. O volume da cavidade interna dos pós secos por atomização foi estimado em quatro frações de tamanho ao se cortar os aglomerados, previamente colados em cartolina, e ao se medir o tamanho relativo do grânulo e a cavidade, também por microscopia óptica.

Foi avaliada a distribuição de tamanho do aglomerado do atomizado das amostras prensadas a 50,75, 150 e $300 \mathrm{kgf} / \mathrm{cm}^{2}$. As amostras foram secas anteriormente em uma câmara para porosimetria por injeção de mercúrio a $2 \mathrm{kbar}$

\section{Ângulo estático de repouso}
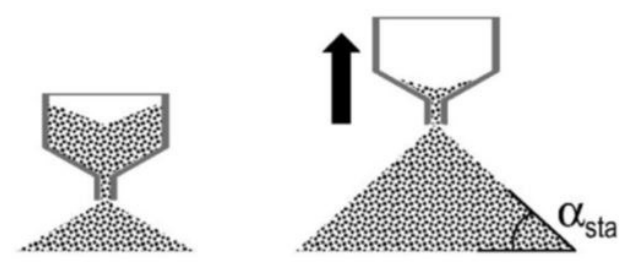

Densidade aparente sem compactar e após compactação

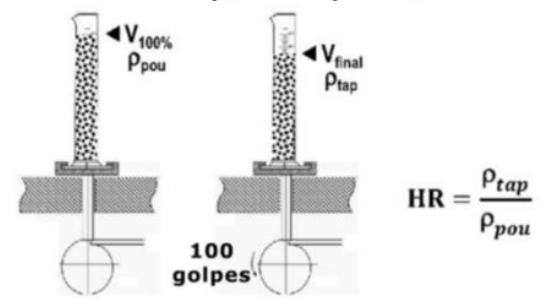

Ângulo dinâmico de repouso

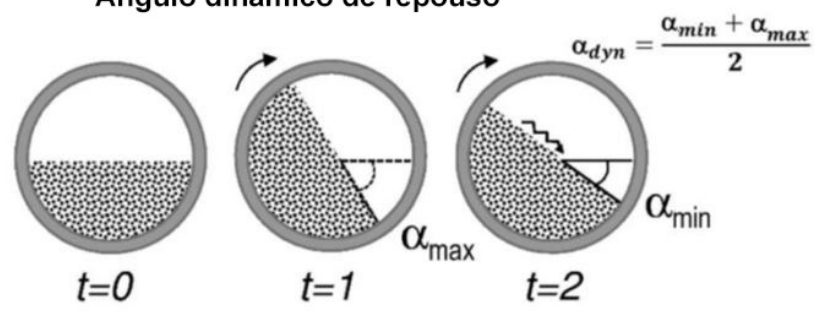

Índice de fluxo mássico

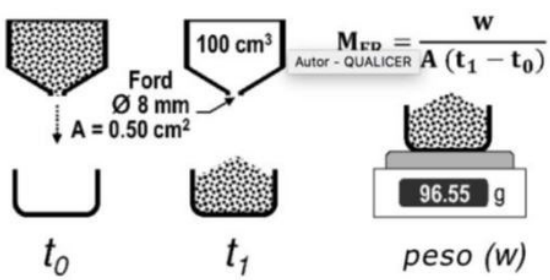

Figura 1. Métodos de determinação das propriedades reológicas dos pós atomizados. 
(correspondente a diâmetro de poro de $\sim 50 \mathrm{~nm}$ ). Além do tamanho de poro, foi determinado o volume de poro (VP), a densidade aparente (DA) e a porosidade aberta. A compressão da amostra $(\mathrm{Cp})$ durante o ensaio, calculada como $\mathrm{C}_{\mathrm{p}}=\mathrm{VP}-[(1$ / DA $)-(1-\mathrm{DR})]$, foi atribuída ao colapso da cavidade interna. A densidade real (DR) dos corpos foi determinada por picnometria a hélio.

\section{Resultados e Discussão}

\subsection{Características dos pós atomizados}

A distribuição de tamanho do aglomerado das amostras de porcelanato é mostrada na Figura 2. Pode-se observar dois tipos de distribuição: pós de grânulo mais fino (tamanho médio de $250 \mu \mathrm{m}$ ) e pós com grânulos maiores (tamanho médio de $350 \mu \mathrm{m}$ ). A contribuição baseia-se na comparação de duas amostras, que são representativas de pós de grânulo mais fino e maior, respectivamente.

A umidade varia segundo o tamanho do aglomerado (Figura 3). Há uma tendência geral do aumento da umidade

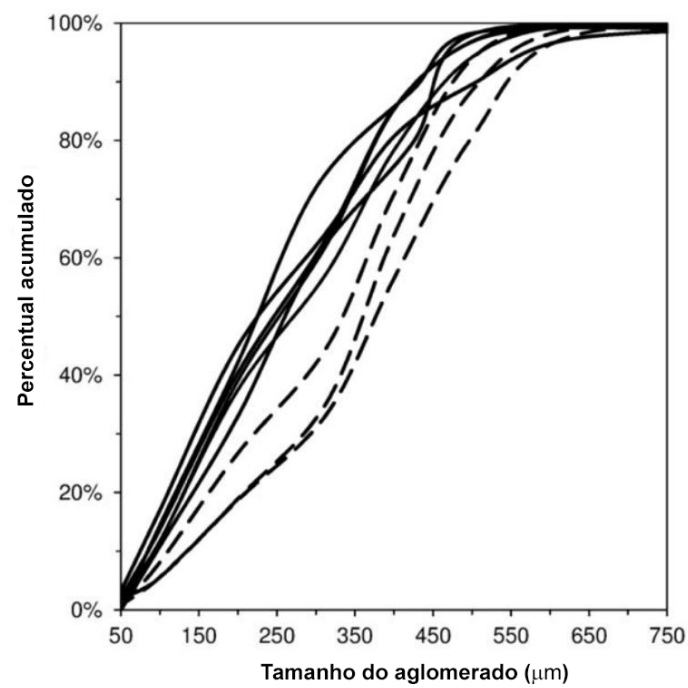

Figura 2. Distribuição de tamanho do aglomerado do porcelanato (linha descontínua = grânulos maiores). com o aumento do tamanho do grânulo. Por esta razão, os pós mais finos são menos úmidos que os maiores (Tabela 1). Apesar de ser uma tendência esperada, os dados permitem determinar a diferença de umidade, p.ex., entre frações de 400-500 $\mu \mathrm{m}$ e 100-200 $\mu \mathrm{m}$, em cerca de $2 \%$ (em valores entre 0,5 e 4,5\%). Há flutuações em frações intermediárias de tamanho e também para distribuição plana, com a máxima umidade na fração mais fina.

Os aglomerados apresentam formas distintas que aparecem com uma frequência variável em frações de distintos tamanhos (Figura 4). Estas formas podem ser atribuídas à morfologia dos grânulos atomizados, mas também a outros casos, como grânulos rompidos ou a coalescência de dois ou mais grânulos (Figura 5). A distribuição da forma varia muito em função do tamanho do aglomerado, mas há uma tendência geral: os grânulos com forma de 'pipoca' aparecem nas frações mais grossas (> $400 \mu \mathrm{m}$ ) e os grânulos 'gêmeos' (morfologias 3 e 4 da Figura 5) são mais frequentes nas frações intermediárias (200 a $500 \mu \mathrm{m})$.

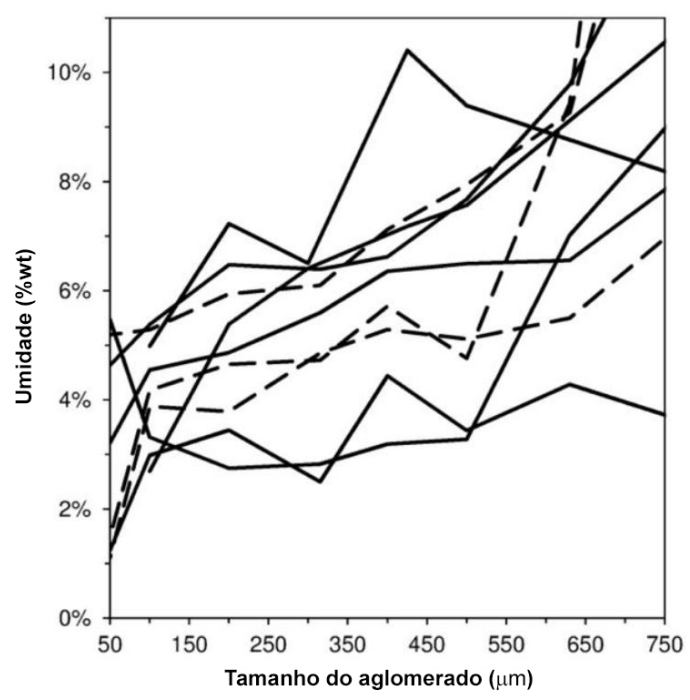

Figura 3. Distribuição da umidade segundo o tamanho do aglomerado do porcelanato.

Tabela 1. Características e comportamento reológico dos pós atomizados.

\begin{tabular}{|c|c|c|c|c|c|c|c|}
\hline \multirow{2}{*}{ Parâmetro } & \multirow{2}{*}{ Unidade } & \multicolumn{3}{|c|}{ Pós de grânulo mais fino } & \multicolumn{3}{|c|}{ Pós de grânulos maiores } \\
\hline & & Mín. & Máx. & Média & Mín. & Máx. & Média \\
\hline Tamanho médio dos aglomerados & $\mu \mathrm{m}$ & 225 & 270 & 247 & 335 & 375 & 358 \\
\hline Aglomerados superiores a $500 \mu \mathrm{m}$ & $\%$ em massa & 2,2 & 15 & 6,4 & 5,8 & 23,1 & 15,8 \\
\hline Aglomerados com forma de 'pipoca' & $\%$ em massa & 4,1 & 36,7 & 16,6 & 15,9 & 34,2 & 28 \\
\hline Umidade dos pós atomizados & $\%$ em massa & 3,2 & 6,1 & 4,8 & 4,5 & 7,2 & 6 \\
\hline Ângulo estático de repouso, $\alpha_{\text {est }}$ & $\circ$ & 25 & 34 & 30 & 30 & 34 & 32 \\
\hline Ângulo dinâmico de repouso, $\alpha_{\text {est }}$ & $\circ$ & 34 & 45 & 39 & 34 & 43 & 38 \\
\hline Índice de fluxo mássico, $\mathrm{I}_{\mathrm{FM}}$ & $\mathrm{g} \cdot \mathrm{cm}^{-2} \cdot \mathrm{s}^{-1}$ & 13,3 & 15,9 & 14,8 & 13,5 & 15 & 14,1 \\
\hline Densidade aparente sem compactação, $\rho_{\mathrm{AP}}$ & $\mathrm{g} \cdot \mathrm{cm}^{-3}$ & 0,93 & 1,01 & 0,98 & 0,95 & 1,05 & 0,98 \\
\hline Densidade aparente após compactação, $\rho_{\text {comp }}$ & $\mathrm{g} \cdot \mathrm{cm}^{-3}$ & 1,03 & 1,12 & 1,1 & 1,04 & 1,12 & 1,1 \\
\hline Índice de Hausner, IH & 1 & 1,09 & 1,17 & 1,12 & 1,07 & 1,15 & 1,12 \\
\hline
\end{tabular}




\begin{tabular}{|c|c|c|c|c|c|c|c|c|}
\hline$<100$ & $100-200$ & $200-315$ & $315-400$ & $400-500$ & $500-630$ & $630-1000$ & $1000-2000$ & 乍 \\
\hline & sis & $8 \mathrm{x} 2$ & 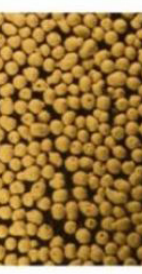 & 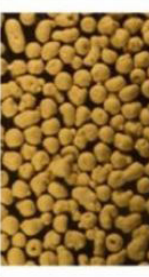 & & & 3 & 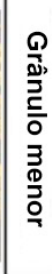 \\
\hline & 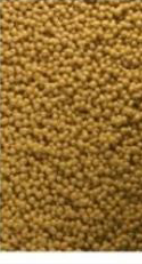 & $\frac{5}{5}$ & tos & 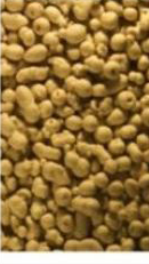 & 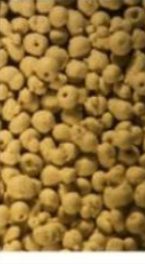 & 8 & 5 & 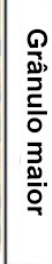 \\
\hline
\end{tabular}

Figura 4. Forma dos grânulos atomizados em frações de diferentes tamanhos.
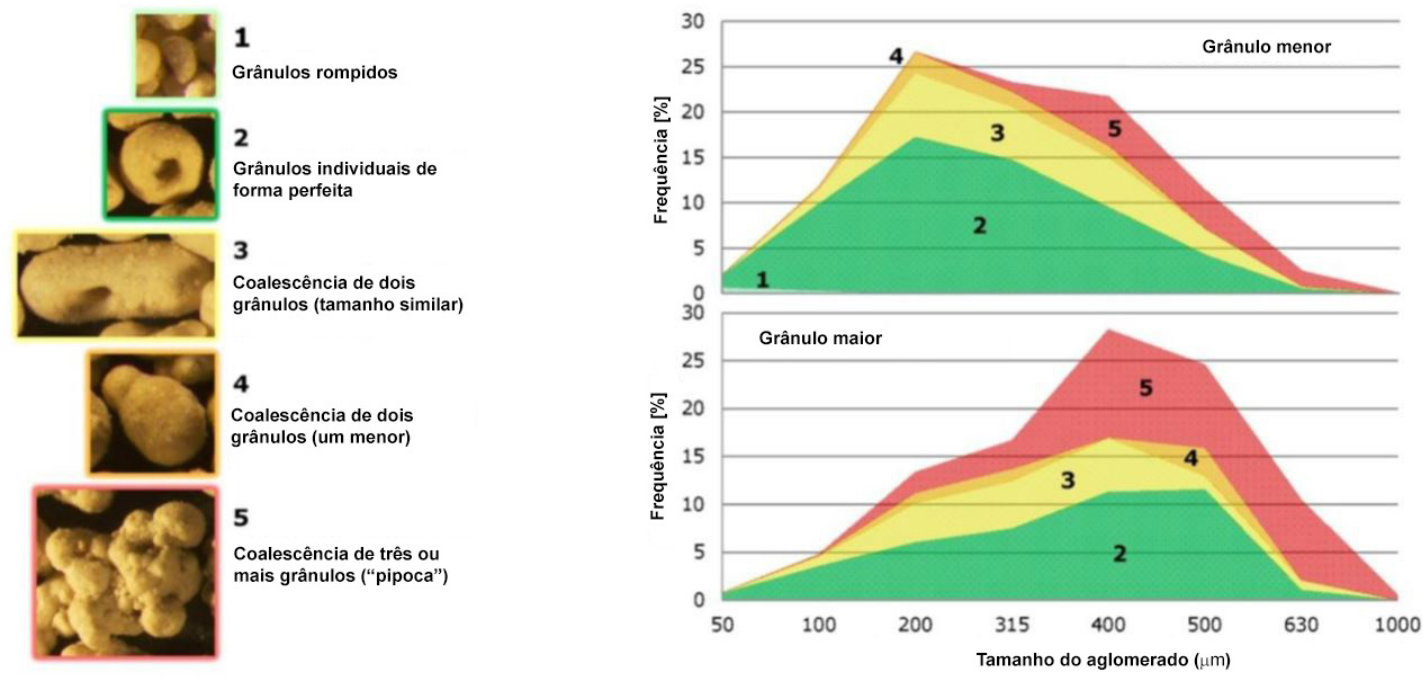

Figura 5. A morfologia do grânulo é definida de acordo com cinco formas básicas (esquerda). Distribuição da forma do aglomerado: exemplos de pós de grânulo mais fino e grânulo maior (direita).

No entanto, esta visão geral descrita na Figura 5 para os pós de grão mais fino e grão maior, não é sistemática. Podem ocorrer mudanças significativas de acordo com o tratamento do pó (secagem por atomização, armazenamento, possível umedecimento ou condensação) [1].

\subsection{Propriedades reológicas dos pós secos por atomização}

Os valores medidos para as propriedades reológicas dos pós de grão mais fino e grão maior são resumidos na Tabela 1. Tanto os ângulos estáticos quanto os dinâmicos de repouso variam em um intervalo bastante estreito ( 25 a $34^{\circ} \alpha_{\text {est }}$ e $34-45^{\circ} \alpha_{\text {din }}$ ), com os valores dos pós de grão mais fino e maior sobrepostos consideravelmente. Este fato dificulta a obtenção de uma diferença clara da fluência pelo ângulo de repouso. A incerteza experimental é de $1^{\circ}$, tendo-se em conta o viés causado pela aparição de um ângulo inferior e um ângulo superior, o que não é uma diferença trivial. Para o caso do ângulo estático de repouso, os valores medidos correspondem a pós que 'fluem livremente' [8]. Os valores muito baixos $\left(<30^{\circ}\right)$, apesar de terem 'fluência excelente', parece que só são alcançáveis por alguns pós atomizados de grão mais fino.

O intervalo de índice de fluxo mássico é praticamente o mesmo (13 a $\left.16 \mathrm{~g} / \mathrm{cm}^{3} . \mathrm{s}\right)$ para pós de grão mais fino e grão maior (Tabela 1) quando se utiliza um orifício relativamente largo ( $8 \mathrm{~mm}$ de diâmetro). O fluxo mássico diminui ao se reduzir o tamanho do orifício (p.ex., 6 ou $4 \mathrm{~mm}$, mas não se pode perceber uma distinção em termos de fluência do pó). 
A densidade aparente sem compactação varia de 0,93 a $1,05 \mathrm{~g} / \mathrm{cm}^{3}$, com um valor de $0,98 \mathrm{~g} / \mathrm{cm}^{3}$ para pós de grão mais fino e grão maior, embora pareça que as maiores densidades são obtidas para os pós mais finos (Tabela 1). Esta determinação simples de aparência é muito afetada pelas condições experimentais (p.ex., altura de queda, geometria do reservatório, velocidade de carregamento) e, portanto, depende muito do analista.

Os pós de grão mais fino e grão maior apresentam o mesmo intervalo de valores $\left(1,03\right.$ a $\left.1,12 \mathrm{~g} / \mathrm{cm}^{3}\right)$ para a densidade compactada (Tabela 1). Porém, são observadas diferentes curvas de empacotamento (Figura 6). A eficiência do empacotamento é dada pela distribuição do tamanho dos aglomerados e, em particular, ao índice favorável de dimensões maiores e mais finas [33,34].

Em geral, o índice de Hausner compreende desde 1,07 a 1,17, e não há uma diferença substancial entre os pós de grão mais fino e de grão maior (Tabela 1). Todos os valores estão abaixo de 1,25, e correspondem a pós que 'fluem livremente' [8]. A maioria dos corpos atomizados apresentam um $\mathrm{IH}<1,15 \mathrm{e}$, portanto, podem ser considerados pós com 'fluência excelente'. Aparentemente, a relação esperada entre o índice de Hausner e o ângulo de repouso não é aplicada aos pós cerâmicos porque os intervalos de variação tanto do IH quanto do $\alpha_{\text {est }}$ são muito pequenos para permitir qualquer tendência clara, como as observadas para um conjunto amplo de pós diferentes (Figura 7).

As características físicas dos pós atomizados contrastam com suas propriedades reológicas em uma matriz de correlações binárias (Figura 8). Algumas relações se destacam com uma tendência similar para o tamanho e a forma do aglomerado, para a fração maior $(>500 \mu \mathrm{m})$ e para o percentual de grânulos com forma de 'pipoca'. São percebidas as mesmas tendências para o tamanho médio de aglomerados, mas sem importância.

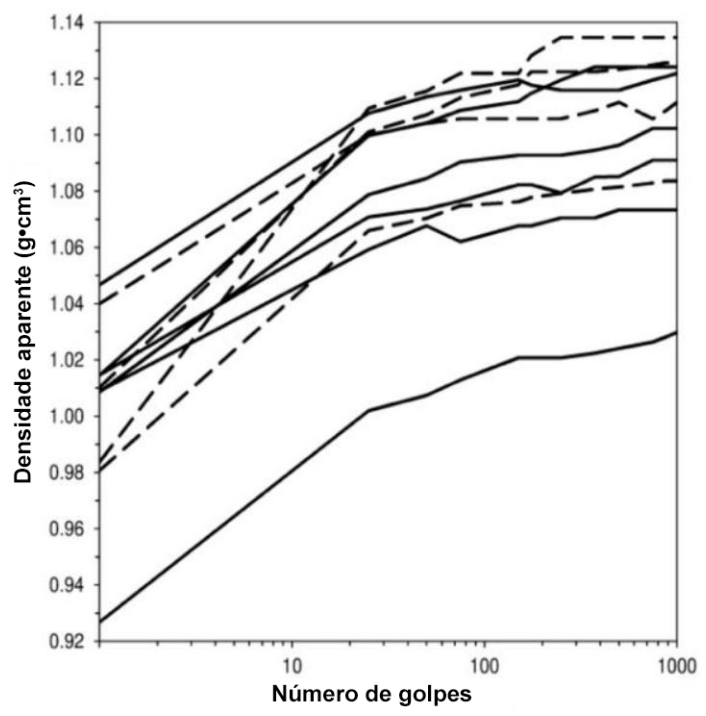

Figura 6. Curvas de densidade aparente segundo os golpes para pós de grão maior (linhas descontínuas) e de grão mais fino.
Tanto os ângulos estáticos quanto os dinâmicos de repouso estão diretamente relacionados com o tamanho e a forma do aglomerado: quanto maior seja a quantidade de grãos maiores e com forma de 'pipoca', maior será $\alpha_{\text {est }}$ e $\alpha_{\text {din }}$. $\mathrm{O}$ índice de fluxo mássico aumenta à medida que o tamanho médio do aglomerado diminui. Esta tendência é claramente dependente do percentual dos grânulos maiores e de forma irregular.

A densidade aparente sem compactar correlaciona-se com as características dos pós atomizados de forma complexa e, por tanto, são observadas as distribuições amplas da Figura 8. De qualquer modo, $\rho_{\text {ap }}$ é mais alta quando a quantidade de grânulos maiores é $<10 \%$. A densidade compactada parece ser independente das características aqui consideradas para os pós atomizadas. No entanto, a eficiência de empacotamento dos grânulos depende dos índices entre frações de tamanhos maiores, finas e intermediárias $[33,34]$, sendo a razão para a elaboração de dados mais profunda para revelar se isto também é válido para os pós cerâmicos. Surgiram tendências mais amplas para o índice de Hausner, em especial uma correlação direta com a quantidade de grânulos maiores e com forma de 'pipoca'.

A umidade não afeta de forma substancial o comportamento reológico dos pós. Talvez a única exceção seja o índice de fluxo mássico, que mostra uma tendência análoga às observadas em comparação com o tamanho e a forma do aglomerado. Esta afirmação é válida só para o intervalo de umidade das amostras pesquisadas. Em geral, as correlações mais significativas são com o percentual de grânulos maiores e com forma irregular: positiva para o índice de Hausner e para o ângulo de repouso, negativa para o índice de fluxo mássico e para a densidade aparente sem compactar. Todas estas tendências convergem até uma fluência inferior dos pós que contêm mais grânulos em forma de 'pipoca' ou aglomerados de mais de $500 \mu \mathrm{m}$.

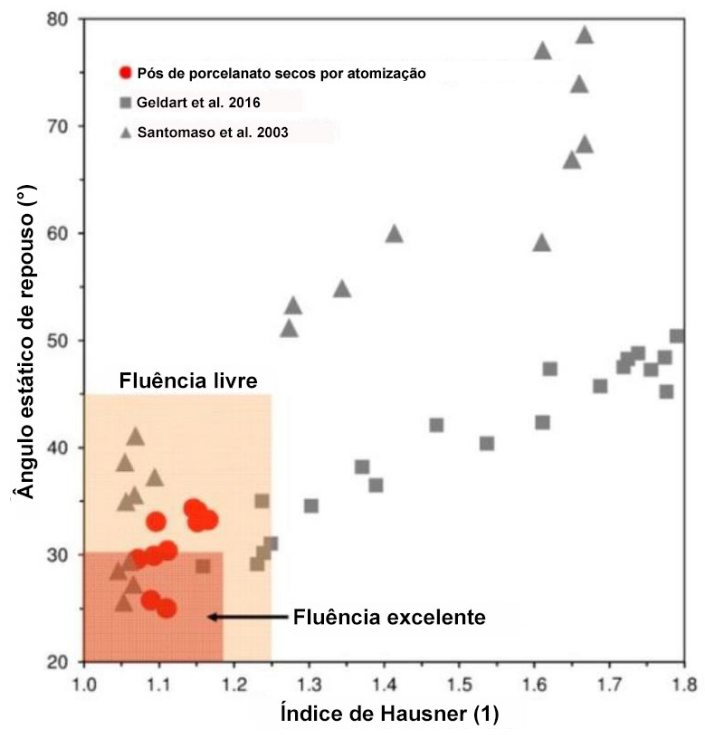

Figura 7. Correlação entre o índice de Hausner e o ângulo estático de repouso dos pós cerâmicos em comparação com outros tipos de pós [8,9]. 


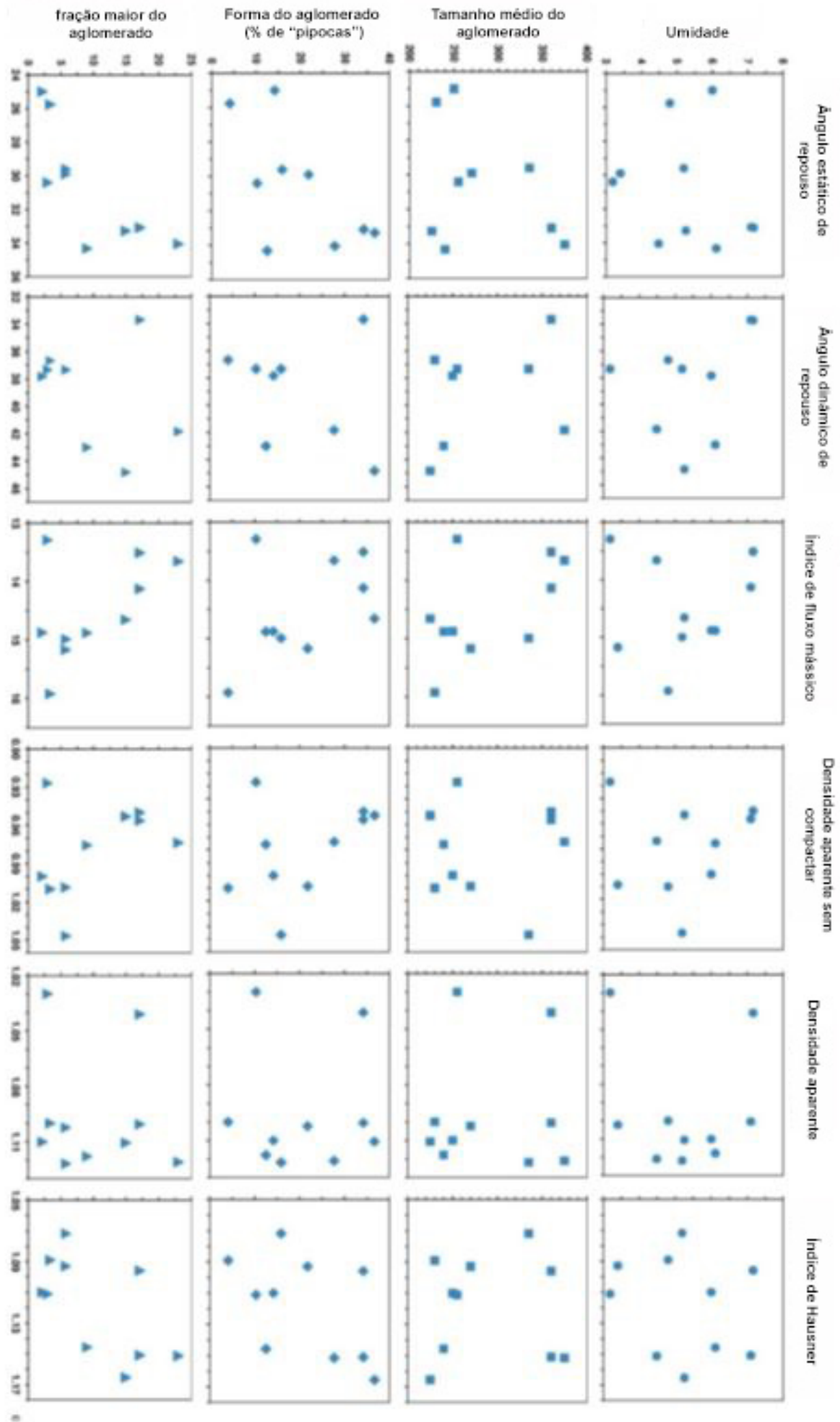

Figura 8. Matriz de correlação das características dos pós atomizados (tamanho do aglomerado, forma e umidade) em relação aos parâmetros reológicos principais (ângulo estático e dinâmico de repouso, índice de fluxo mássico, densidade aparente sem compactar e compactada, índice de Hausner). 
Esta observação tem repercussões na modelagem da disposição espacial dos pós atomizados [35,36]. O desvio principal da predição está relacionado com a aparição de agregados irregulares e de maior tamanho.

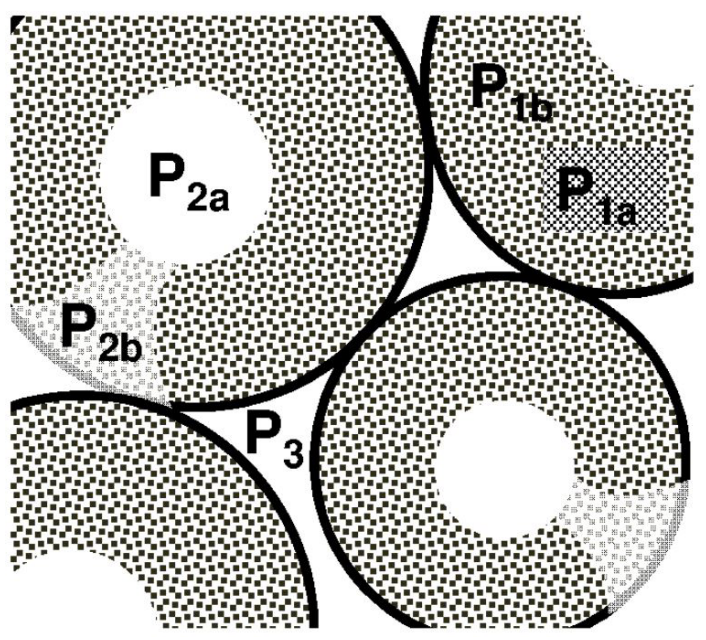

Figura 9. Tipos de porosidade na camada mais suave do pó. Microporosidade intragranular: incompressível $\left(\mathrm{P}_{1 \mathrm{a}}\right)$ e compressível $\left(\mathrm{P}_{1 b}\right)$; macroporosidade intragranular: cavidade $\left(\mathrm{P}_{2 \mathrm{a}}\right)$ e orifício $\left(\mathrm{P}_{2 \mathrm{~b}}\right)$ : porosidade intergranular $\left(\mathrm{P}_{3}\right)$.

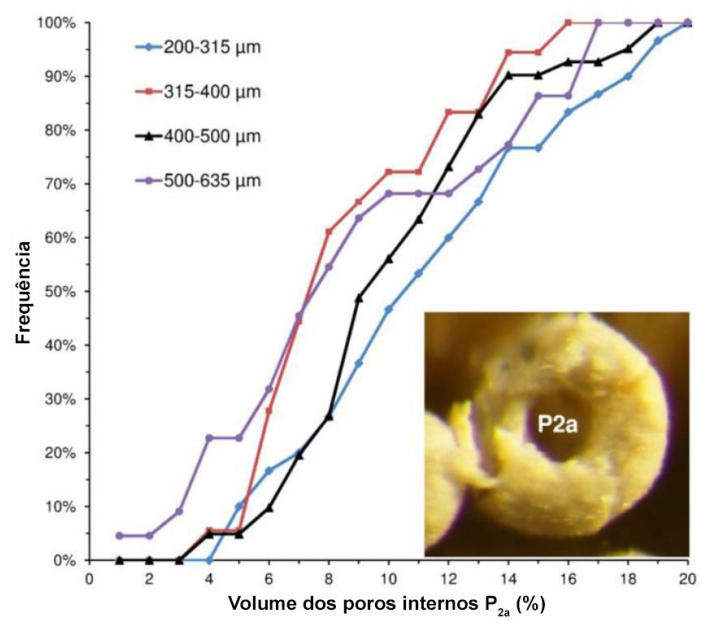

Figura 10. Volume relativo de porosidade intragranular P2a em grânulos de diferentes tamanhos.

\subsection{Comportamento de compactação dos pós atomizados}

Se vislumbra uma situação inicial à medida que os aglomerados enchem uma matriz ou se depositam em uma camada suave sobre uma esteira (Figura 9). Podem ser percebidos distintos tipos de porosidade: intergranular e intergranular 8tanto microporosidade como macroporosidade). A microporosidade intergranular é representada por poros de tamanho micrométrico, que podem ser incompressíveis $\left(\mathrm{P}_{1 \mathrm{a}}\right)$ ou compressíveis $\left(\mathrm{P}_{1 \mathrm{~b}}\right)$ sob as pressões aplicadas normalmente na fabricação de placas. A macroporosidade intragranular é característica dos grânulos atomizados. consiste em uma cavidade quase esférica $\left(\mathrm{P}_{2 \mathrm{a}}\right)$ e um orifício $\left(\mathrm{P}_{2 \mathrm{~b}}\right)$ que conecta a cavidade com a superfície externa. A porosidade intergranular (P3) depende da eficiência de empacotamento dos grânulos durante sua deposição na esteira ou em uma matriz.

$\mathrm{O}$ volume relativo da porosidade intragranular $\left(\mathrm{P}_{2 \mathrm{a}}\right)$ é em média $10 \%$ do volume dos grânulos, independentemente do tamanho dos aglomerados, com quase todos os valores entre 5 e 15\% (Figura 10). Estes dados são coerentes com estimativas anteriores [1,24], embora as estimativas somente são válidas em casos de morfologia ideal dos grânulos atomizados.

A compactação de pós atomizados com uma pressão crescente ocasiona uma alteração clara na microestrutura. A porosidade intergranular $\left(\mathrm{P}_{3}\right)$ fecha progressivamente $\mathrm{e}$ os grânulos individuais se deformam para formar uniões triplas, onde alguns poros residuais persistem acima de $150 \mathrm{kgf} / \mathrm{cm}^{2}$ (Figura 11).

As curvas de densidade aparente produzidas em comparação com a pressão mostram os regimes de compactação: o primeiro, uma densidade que aumenta rapidamente até aproximadamente $13 \mathrm{MPa}$, seguida de um ganho mais lento de densidade a pressões mais altas (Figura 12a). A aparição de uma mudança abrupta entre os dois regimes só é aparente (pois não foi possível compilar dados abaixo de $13 \mathrm{MPa}$ no ensaio com prensa de laboratório). Na verdade, há uma zona de transição gradual quando se faz o ensaio de uma única vez (quadro na Figura 12a).

As curvas parecem corresponder ao comportamento de prensagem do pó atomizado eu contêm agregados que podem romper-se durante a compactação [21]. Os pós de grão maior tendem a se compactar mais que os de grão mais fino, embora hajam exceções (Figura 12b), o que
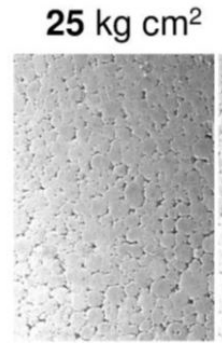

50

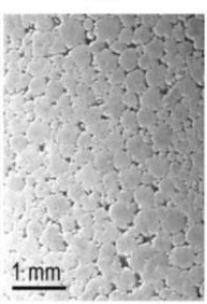

75

100

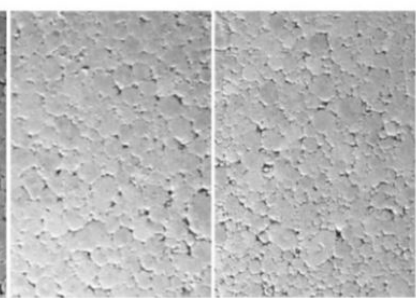

150

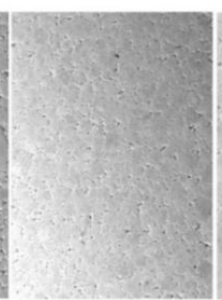

200

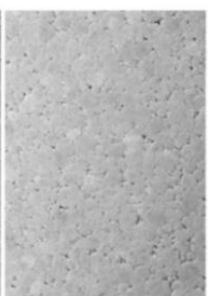

300

Figura 11. Microestrutura da superfície dos compactos prensados a pressões crescentes. 

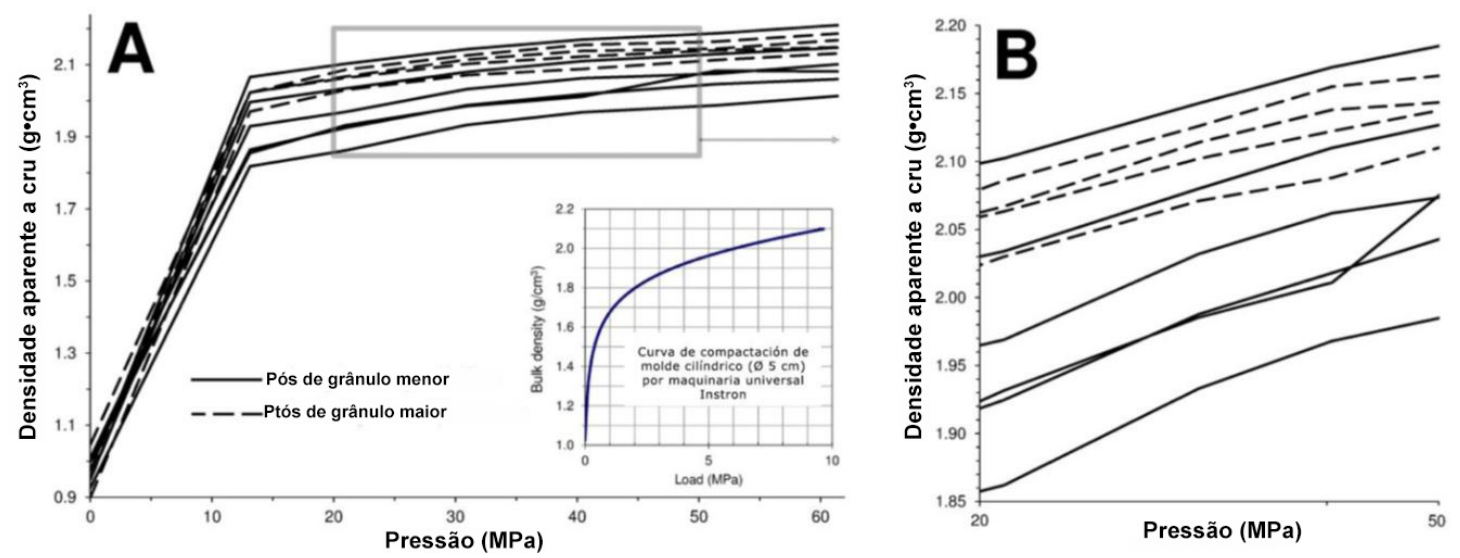

Figura 12. Curvas de compactação de pós atomizados para amostras preparadas em uma prensa de laboratório em comparação com amostras preparadas uma única vez (A); comparação detalhada com pressão em escala logarítmica (B).

está relacionado com a diferente distribuição de umidade, pois os pós de grão maior são mais úmidos.

A compressibilidade dos pós atomizados, expressa pelo índice de Carr a $40 \mathrm{MPa}$ de pressão, é de 50 a $58 \%$, com a maioria das amostras entre 50 e $55 \%$. Os dois regimes de compactação podem se relacionar com o trabalho necessário para comprimir os distintos tipos de porosidade descritos na Figura 1. A evolução da porosidade em pressão crescente foi obtida de forma quantitativa por porosimetria de mercúrio: as curvas cumulativas e os histogramas de distribuição de tamanho de poro são mostradas na Figura 13 para o regime baixo de $\mathrm{P}$ $\left(50\right.$ e $\left.75 \mathrm{kgf} / \mathrm{cm}^{2}\right)$, o regime alto $\left(300 \mathrm{kgf} / \mathrm{cm}^{2}\right)$ e a zona de transição $\left(150 \mathrm{kgf} / \mathrm{cm}^{2}\right)$.

Ao se comparar as curvas de tamanhos de poros pode-se observar uma redução progressiva dos macroporos (quer dizer, a população de poros entre 3 e $15 \mu \mathrm{m}$, com um máximo de $8 \mu \mathrm{m}$ ) devido a uma pressão crescente de 50 a $150 \mathrm{kgf} / \mathrm{cm}^{2}$, e praticamente desapareceram. Esta população de poros é atribuída à porosidade intergranular $\mathrm{P}_{3}$. Seu fechamento gradual corresponde à inclinação pronunciada das curvas de compactação (o regime de baixo $\mathrm{P}$ ).

O amplo intervalo de microporos presente nos grânulos (que vai desde $3 \mu \mathrm{m}$ a $50 \mathrm{~nm}$ ) se deforma gradualmente em pressões mais altas. Desta forma, os microporos maiores, que variam aproximadamente de 3 a $0,7 \mu \mathrm{m}$, são completamente reduzidos a $300 \mathrm{kgf} / \mathrm{cm}^{2}$. Isto se reflete na menor inclinação das curvas de compactação acima de $150 \mathrm{kgf} / \mathrm{cm}^{2}$ (o regime alto de P).

A evolução dos distintos tipos de porosidade na camada mais suave dos pós atomizados pode ser descrita ao se comparar os dados da porosimetria de mercúrio, densidade aparente sem compactar e microscopia óptica (Figura 14). A porosidade intergranular $\mathrm{P}_{3}$ fecha rapidamente nas fases iniciais da compactação e desaparece quase por completo a $150 \mathrm{kgf} / \mathrm{cm}^{2}$. A cavidade interna some aparentemente a uma pressão muito baixa $\left(<50 \mathrm{kgf} / \mathrm{cm}^{2}\right)$.

Percebe-se em alta pressão uma porosidade residual $\mathrm{P}_{3}$ atribuída ao orifício. A maior compactação com pressão desenvolve-se pela redução do tamanho da porosidade; em primeiro, $\mathrm{P}_{2}$ e $\mathrm{P}_{3}$, que ocasionam um aumento da fração maior de microporos $\left(\mathrm{P}_{1 \mathrm{~b}}\right)$ a $50 \mathrm{kgf} / \mathrm{cm}^{2}$. Com o aumento da pressão, os microporos compressíveis $\left(\mathrm{P}_{1 b}\right)$ convertem-se progressivamente na fração incompressível $\left(\mathrm{P}_{1 \mathrm{a}}\right)$. A pressão atua simultaneamente sobre os distintos tipos de porosidade: $\mathrm{P}_{2 \mathrm{a}}$ e $\mathrm{P}_{3}$ são fechadas (e até certo ponto reduzidas a $\mathrm{P}_{1 b}$ ) no regime baixo de $\mathrm{P}$, enquanto no regime alto de $\mathrm{P}$ os poros compressíveis $\left(\mathrm{P}_{1 b}\right.$ e talvez o orifício $\mathrm{P}_{2 \mathrm{~b}}$ ) reduzem-se a $\mathrm{P}_{1 \mathrm{a}}$.

A saturação dos poros por água pode ser estimada ao se assumir que a umidade inicial do pó atomizado está contida essencialmente na microporosidade e retida em sua totalidade durante a prensagem. Neste caso, a saturação inicial do poro (volume ocupado por água do volume total de microporos) situa-se entre 19 e $21 \%$ (para pós atomizados com umidade de 6 a 7\%). Este grau de saturação aumenta entre 23 e $25 \%$ na placa prensada a $300 \mathrm{kgf} / \mathrm{cm}^{2}$ e aumentará algo mais na pressão industrial $\left(\sim 400 \mathrm{kgf} / \mathrm{cm}^{2}\right)$.

Estes fatos levam implicações para o modelo de compactação: deve ter-se em conta uma alteração, com uma pressão crescente, na reação mecânica dos grânulos atomizados. De fato, os aglomerados tornam-se mais e mais duros quando a porosidade intergranular e sua cavidade interna se fecham e quando se realiza trabalho para reduzir o tamanho da microporosidade compressível.

Pode estar presente um grau diferente de compactação em placas cruas, por exemplo, à medida que os valores da densidade aparente a seco mudam ponto a ponto. As diferenças na densidade aparente a seco diminuem durante a queima, pois um corpo mais poroso sinteriza mais rapidamente que um menos poroso [37]. Este fenômeno pode ser observado quando são considerados dois pós atomizados (de grão mais fino e grão maior, respectivamente, quer dizer, os dois mais densos na Figura 12) que foram compactados a pressões crescentes de 13 a $80 \mathrm{MPa}$. Foi obtido um intervalo de densidade aparente a seco de 1,9 a $2,03 \mathrm{~g} / \mathrm{cm}^{3}$ (corpo de grão maior) e de $1,94 \mathrm{a} 2,08 \mathrm{~g} / \mathrm{cm}^{3}$ (corpo de grão mais fino); o intervalo geral é de 0,13 a $0,14 \mathrm{~g} / \mathrm{cm}^{3}$. 


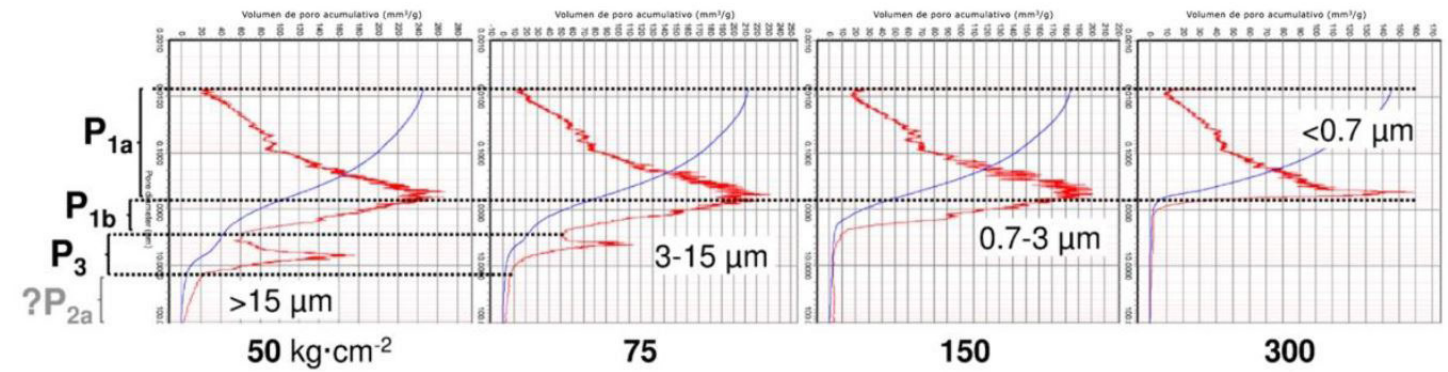

Figura 13. Curvas de distribuição de tamanho de poro (volume de poro e cumulativo por porosimetria de mercúrio) segundo a pressão (de 50 a $300 \mathrm{kgf} / \mathrm{cm}^{2}$ ). Os tipos de poros estão relacionados com os intervalos de tamanhos de poros.
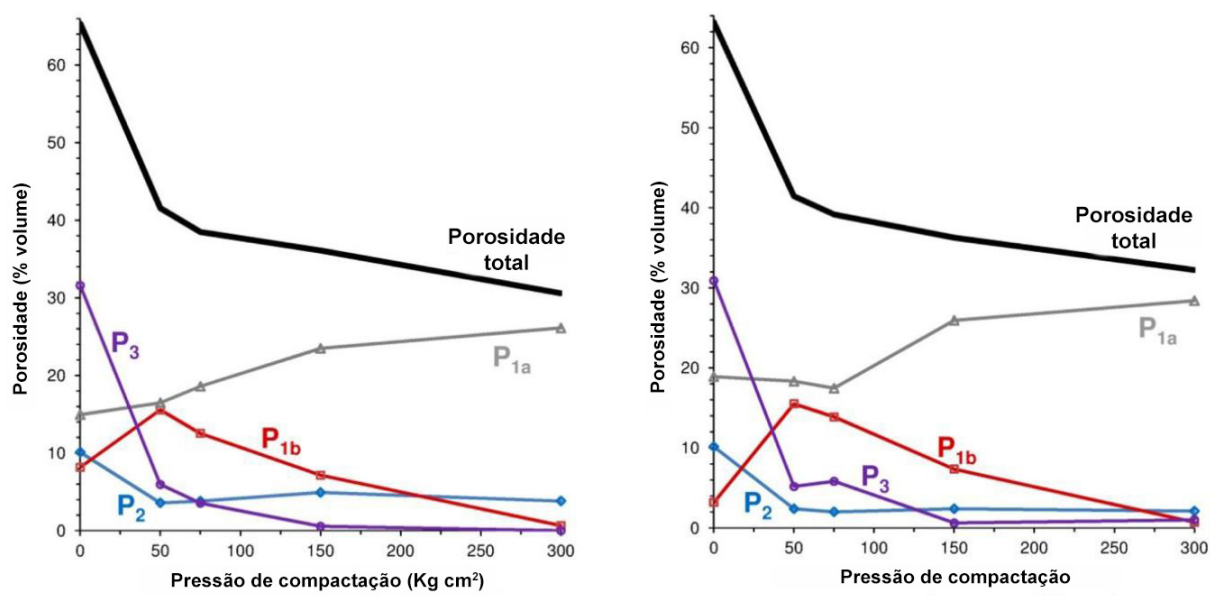

Figura 14. Variação de diferentes tipos de porosidade da camada mais suave de pós atomizados (Fig. 9) em função da pressão sobre pós de grão mais fino (esquerda) e pós de grão maior (direita).

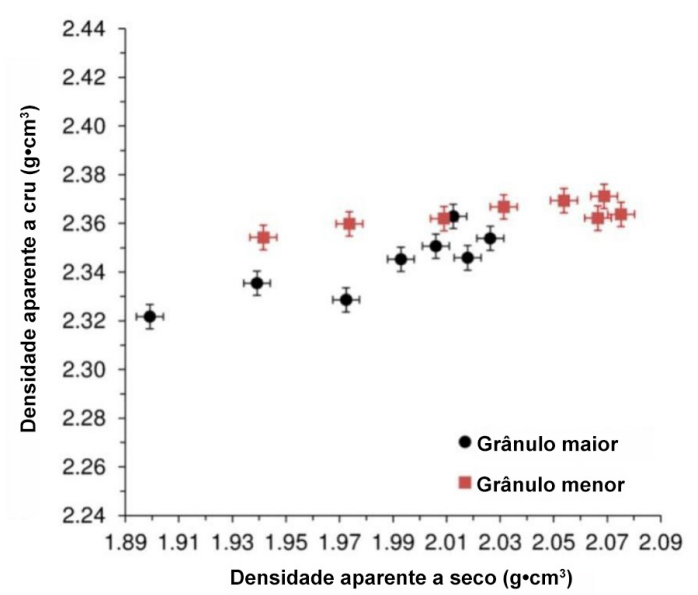

Figura 15. Densidade aparente de placas de porcelanato não queimadas, prensadas de 13 a $80 \mathrm{MPa}$. São comparados corpos com diferente distribuição de tamanho de aglomerado.

Estes valores contrastam com a densidade aparente das amostras correspondentes, queimadas com um programa industrial, representando os dados com a mesma escala (Figura 15). Os corpos reduziram durante a queima e a densidade aparente mais alta obtida foi de 2,32 a $2,36 \mathrm{~g} / \mathrm{cm}^{3}$ (corpo de grão maior) e de 2,35 a 2,37 g/ $/ \mathrm{cm}^{3}$ (corpo de grão mais fino). $\mathrm{O}$ intervalo geral é de 0,02 a $0,04 \mathrm{~g} / \mathrm{cm}^{3}$. Com uma densidade aparente a seco mais baixa, a retração de queima é maior.

Se a diferença de densidade aparente de duas partes de uma mesma peça não queimada é expressa como $100 \%$, esta diferença é de apenas 15 a $30 \%$ depois da queima. Portanto, de 70 a $85 \%$ da diferença inicial é recuperada em uma retração diferente durante o processo de sinterização. Isto implica que qualquer diferença na densidade aparente a seco é compensada em termos de retração diferencial, que pode ser perigosa, e ser a causa provável de uma deformação permanente e de tensões residuais depois da queima [38-40].

\section{Conclusões}

Em geral, as características intrínsecas de pós secos por atomização utilizados para fabricação de placas grandes variam consideravelmente, sendo definidas duas classes em relação à distribuição de tamanho de aglomerado. Os corpos com grãos maiores têm, em média, uma maior quantidade de umidade e grânulos de forma irregular quando são comparados com pós de grão mais fino.

Os corpos atomizados se comportam como pós que fluem livremente durante o carregamento da matriz ou 
a deposição sobre a esteira. São caracterizados por um ângulo estático de repouso bastante estreito $\left(25 \mathrm{a} 34^{\circ}\right)$, um índice de Hausner $(1,07$ a 1,17) e um fluxo mássico $\left(13 \mathrm{a} 15 \mathrm{~g} / \mathrm{cm}^{3} . \mathrm{s}\right)$. As propriedades reológicas não parecem estar relacionadas, devido ao estreito intervalo dos dados e da incerteza experimental, com alto grau de subjetividade nas medições reológicas.

As características intrínsecas dos pós afetam a sua fluência pela aparição de agregados maiores, em particular os de forma irregular, que são produzidos pela coalescência de dois ou mais aglomerados individuais. Por exemplo, quanto menor for a quantidade de grânulos com forma de 'pipoca', melhor será a fluência do pó.

Estas observações põem em evidência a importância do controle de qualidade durante a gestão da atomização e do pó. A formação de agregados maiores deve ser evitada e, desta forma, prevenir a condensação de vapor de água e de gotas na atomização, além de gotas no transporte e armazenamento. É necessária atenção especial nas operações de umedecimento.

A compactação de grânulos atomizados determina uma alteração contínua na quantidade e tipologia dos poros presentes na peça crua. As distintas inclinações das curvas de compactação refletem o trabalho necessário para comprimir, inicialmente, os poros intergranulares $\left(\mathrm{P}_{3}\right)$ e a cavidade interna dos aglomerados $\left(\mathrm{P}_{2 \mathrm{a}}\right)$ e, em seguida, a microporosidade intragranular maior $\left(\mathrm{P}_{1 b}\right)$ e o canal interno dos aglomerados $\left(\mathrm{P}_{2 \mathrm{~b}}\right)$. Só está presente a microporosidade intragranular 'incompressível' $\left(\mathrm{P}_{1 \mathrm{a}}\right)$ nas placas prensadas. $\mathrm{O}$ regime baixo de $\mathrm{P}$ (abaixo de $\sim 10 \mathrm{MPa}$ ) desempenha uma compressão mais rápida (aproximadamente $80 \%$ do aumento de densidade aparente), enquanto que o regime ato de $\mathrm{P}$ atua de forma progressiva com um aumento lento da densidade aparente.

Os corpos de porcelanato utilizados para fabricar placas cerâmicas grandes apresentam uma compressibilidade similar (índice de Carr no intervalo de 50 a 55\%). Embora haja uma variância limitada de forma global, deve-se ter em conta que o grau de compactação depende principalmente do teor de umidade. Todos os corpos têm uma distribuição de umidade em função do tamanho do aglomerado, o que implica que a densidade aparente pode variar na escala da dimensão do aglomerado, particularmente para o caso de grandes grânulos de forma irregular.

As diferenças de densidade aparente das placas não queimadas não se mantêm no produto acabado, pois as peças cruas menos densas sinterizam mais rápido que as de menor densidade. Isto significa que qualquer diferença de densidade aparente a cru converte-se em uma retração diferente durante a queima. Estas retrações diferenciais são a principal causa das deformações permanentes e da tensão residual nas placas grandes.

\section{Agradecimentos}

Os autores agradecem à região da Emilia-Romagna pelo apoio financeiro ao projeto IPERCER "Process innovation for the value chain of the sustainable ceramic tile" (CUP E32I16000010007), POR FESR 2014-2020 (Eje 1, Acción 1.2.2).

\section{Referências}

[1] García-Ten, J., Sánchez, E., Mallol, G., Jarque, J. C., \& Arroyo, A. (2004). Influence of operating variables on spray-dried granule and resulting tile characteristics. In Key Engineering Materials, 264, 1499-1502.

[2] Amorós, J. L., Cantavella, V., Jarque, J. C., \& Felíu, C. (2008). Fracture properties of spray-dried powder compacts: effect of granule size. Journal of the European Ceramic Society, 28(15), 2823-2834.

[3] Raimondo, M., Dondi, M., Zanelli, C., Guarini, G., Gozzi, A., Marani F., \& Fossa, L. (2010). Boletín de la Sociedad Española de Cerámica y Vidrio, 49 (4), 289-295.

[4] Bresciani, A., \& Ricci, C. (2008). Technologie de pressage Continua ${ }^{\circledR}$. L'Industrie Céram. \& Verrière, 1016, 24-28.

[5] Romagnoli, M., \& Bignami, F. (2006). Study of the effect of additives on the flowability of ceramic powders. In Qualicer 2006. IX World Congress on Ceramic Tile Quality. (Vol. 3).

[6] Nebelung, M., \& Lung., B. (2009). Flowability of ceramic bulk materials part 1: methods. Ceramic Forum International, 86, E35-E40.

[7] Amorós, J. L., Mallol, G., Feliu, C., \& Orts, M. J. (2011). Study of the rheological behaviour of monomodal quartz particle beds under stress. A model for the shear yield functions of powders. Chemical engineering science, 66(18), 4070-4077.

[8] Santomaso, A., Lazzaro, P., \& Canu, P. (2003). Powder flowability and density ratios: the impact of granules packing. Chemical Engineering Science, 58(13), 2857-2874.

[9] Geldart, D., Abdullah, E. C., Hassanpour, A., Nwoke, L. C., $\&$ Wouters, I. (2006). Characterisation of powder flowability using measurement of angle of repose. China Particuology, 4(3-4), 104-107.

[10]Zhou, Y. C., Xu, B. H., Yu, A. B., \& Zulli, P. (2002). An experimental and numerical study of the angle of repose of coarse spheres. Powder technology, 125(1), 45-54.

[11] Wong, A. C. Y. (2002). Use of angle of repose and bulk densities for powder characterisation and the prediction of minimum fluidisation and minimum bubbling velocities. Chemical engineering science, 57(14), 2635-2640.

[12] Mellmann, J. (2001). The transverse motion of solids in rotating cylinders - forms of motion and transition behavior. Powder Technology, 118(3), 251-270.

[13]Abdullah, E. C., \& Geldart, D. (1999). The use of bulk density measurements as flowability indicators. Powder technology, 102(2), 151-165.

[14] Mallol, G., Amoros, J. L., Orts, M. J., \& Llorens, D. (2008). Densification of monomodal quartz particle beds by tapping. Chemical Engineering Science, 63(22), 5447-5456.

[15] Amorós, J. L., Orts, M. J., Garcia-Ten, J., Gozalbo, A., \& Sánchez, E. (2007). Effect of the green porous texture on porcelain tile properties. Journal of the European Ceramic society, 27(5), 2295-2301.

[16]Reed, J. S. (2000). From batch to pressed tile: mechanics and system microstructural changes. Qualicer 2000, 1, Con23-42.

[17] Alves, H. J., Melchiades, F. G., \& Boschi, A. O. (2010). Effect of spray-dried powder granulometry on the porous microstructure of polished porcelain tile. Journal of the European Ceramic Society, 30(6), 1259-1265.

[18]Zanelli C., Raimondo M., Guarini G., Marani F., Fossa L., Dondi M., Porcelain stoneware large slabs: processing and 
technological properties. Proc. 11th World Congress on Ceramic Tile Quality, QUALICER 2010, Castellón (Spain), p. 10.

[19] da Silva, A. L., Feltrin, J., Dal Bó, M., Bernardin, A. M., \& Hotza, D. (2014). Effect of reduction of thickness on microstructure and properties of porcelain stoneware tiles. Ceramics International, 40(9), 14693-14699.

[20] Lukasiewicz, S. J. (1989). Spray-Drying Ceramic Powders. Journal of the American Ceramic Society, 72(4), 617-624.

[21] Niesz, D. E. (1996). A review of ceramic powder compaction. KONA Powder and Particle Journal, 14, 44-51.

[22] Beltrán, V., Ferrer, C., Bagán, V., Sánchez, E., Garcia, J., \& Mestre, S. (1996). Influence of pressing powder characteristics and firing temperature on the porous microstructure and stain resistance of porcelain tile. IV World Congress on Ceramic Tile Quality, Castellon, Spain, Vol. 10, No. 13.

[23] SACMI (2001). Ceramic Technology. Editrice La Mandragora, Imola, ISBN 88-88108-16-5.

[24] Gil, C., Silvestre, D., García Ten, F. J., Quereda, M. F., \& Vicente, M. J. (2012). Preparation of porcelain tile granulates by more environmentally sustainable processes. Bol. Soc. Esp. Ceram. Vidr. Vol 51. 2, 67-74,

[25] Shu, Z., Garcia-Ten, J., Monfort, E., Amoros, J. L., Zhou, J., \& Wang, Y. X. (2012). Cleaner production of porcelain tile powders. Granule and green compact characterisation. Ceramics International, 38(1), 517-526.

[26] Kennedy, T., Hampshire, S., \& Yaginuma, Y. (1997). A study of the compaction mechanism of granulated materials. Journal of the European Ceramic Society, 17(2-3), 133-139.

[27] Liu, D. M., Lin, J. T., \& Tuan, W. H. (1999). Interdependence between green compact property and powder agglomeration and their relation to the sintering behaviour of zirconia powder. Ceramics international, 25(6), 551-559.

[28] Sanchez, E., Garcia, J., Barba, A., \& Feliu, C. (2000). Effet de la composition des carreaux porcelainés sur le comportement au pressage de la poudre séchée par atomisation. L'Industrie Céramique \& Verrière, (962), 539-541.

[29] Celik, H. (2011). Effect of spray-dried powder granularity on porcelain tile properties. Journal of Ceramic Processing Research, 12(4), 483-487.

[30] Shu, Z., Garcia-Ten, J., Monfort, E., Amoros, J. L., Zhou, J., \& Wang, Y. X. (2012). Cleaner production of porcelain tile powders. Fired compact properties. Ceramics International, 38(2), 1479-1487.
[31]Pérez, J. M., Rincón, J. M., \& Romero, M. (2012). Effect of moulding pressure on microstructure and technological properties of porcelain stoneware. Ceramics International, 38(1), 317-325.

[32] Santos-Barbosa, D., Hotza, D., Boix, J., \& Mallol, G. (2013). Modelling the influence of manufacturing process variables on dimensional changes of porcelain tiles. Advances in Materials Science and Engineering, 2013, ID 142343, 12 p.

[33]Liu, S., \& Ha, Z. (2002). Prediction of random packing limit for multimodal particle mixtures. Powder technology, 126(3), 283-296.

[34] Liu, D. M., \& Lin, J. T. (1999). Influence of ceramic powders of different characteristics on particle packing structure and sintering behaviour. Journal of materials science, 34(8), 1959-1972.

[35] Tiscar, J. M., Escrig, A., Mallol, G., Pascual, N., Gilabert, F. A., Bonaque, R., \& Pérez, J. A. (2016). Simulation of powder rheological behaviour in the ceramic tile pressing process. Qualicer 2016, XIV Congreso Mundial de la Calidad del Azulejo y del Pavimento Cerámico, Castellón, p. 1-13.

[36] Tiscar, J. M., Escrig, A., Mallol, G., Boix, J., \& Gilabert Villegas, F. A. (2016). Influence of the die filling parameters during the ceramic tile pressing process via Discrete Element Method (DEM). 6th International Conference on Shaping of Advanced Ceramics (Shaping-VI).

[37]Zanelli, C., Ardit, M., Conte, S., Soldati, R., Cruciani, G., Dondi M. (2018). Viscous flow sintering of porcelain stoneware revisited. Proc. 15th World Congress on Ceramic Tile Quality, QUALICER 2018, Castellón (Spain), p. 9.

[38]Cantavella Soler, V., García Ten, F. J., Sánchez Vilches, E. J., Bannier, E., Sánchez, J., Sales, J. (2008). Delayed curvatures in porcelain tiles: analysis and measurement of incluencing factors. Proc. 10th World Congress on Ceramic Tile Quality, QUALICER 2008, Castellón (Spain).

[39] Bannier, E., García-Ten, J., Castellano, J., \& Cantavella, V. (2013). Delayed curvature and residual stresses in porcelain tiles. Journal of the European Ceramic Society, 33(3), 493501.

[40] Melchiades, F. G., Boschi, A. O., dos Santos, L. R., Dondi, M., Zanelli, C., Paganelli, M., \& Mercurio, V. (2014). An insight into the pyroplasticity of porcelain stoneware tiles. Proc. 13th World Congress on Ceramic Tile Quality, QUALICER 2018, Castellón (Spain), p. 11. 